Article

\title{
WEE1 Inhibition Enhances Anti-Apoptotic Dependency as a Result of Premature Mitotic Entry and DNA Damage
}

\author{
Mathilde Rikje Willemijn de Jong ${ }^{1,2}$, Myra Langendonk ${ }^{1,2}{ }^{,}$Bart Reitsma ${ }^{1}$, Pien Herbers ${ }^{1}$, \\ Marcel Nijland ${ }^{1}{ }^{\circledR}$, Gerwin Huls ${ }^{1}$, Anke van den Berg ${ }^{2}{ }^{\circledR}$, Emanuele Ammatuna ${ }^{1}$, Lydia Visser ${ }^{2}$ \\ and Tom van Meerten ${ }^{1, *}$ \\ 1 Department of Hematology, University Medical Center Groningen, University of Groningen, 9713 GZ \\ Groningen, the Netherlands; m.r.w.de.jong@umcg.nl (M.R.W.d.J.); m.langendonk@umcg.nl (M.L.); \\ a.a.reitsma@umcg.nl (B.R.); pienherbers@live.nl (P.H.); m.nijland@umcg.nl (M.N.); g.huls@umcg.nl (G.H.); \\ e.ammatuna@umcg.nl (E.A.) \\ 2 Department of Pathology and Medical Biology, University Medical Center Groningen, University of \\ Groningen, 9713 GZ Groningen, the Netherlands; a.van.den.berg01@umcg.nl (A.v.d.B.); \\ l.visser@umcg.nl (L.V.) \\ * Correspondence: t.van.meerten@umcg.nl; Tel.: +31-503-611-761
}

Received: 27 September 2019; Accepted: 4 November 2019; Published: 7 November 2019

\begin{abstract}
Genomically unstable cancers are dependent on specific cell cycle checkpoints to maintain viability and prevent apoptosis. The cell cycle checkpoint protein WEE1 is highly expressed in genomically unstable cancers, including diffuse large B-cell lymphoma (DLBCL). Although WEE1 inhibition effectively induces apoptosis in cancer cells, the effect of WEE1 inhibition on anti-apoptotic dependency is not well understood. We show that inhibition of WEE1 by AZD1775 induces DNA damage and pre-mitotic entry in DLBCL, thereby enhancing dependency on BCL-2 and/or MCL-1. Combining AZD1775 with anti-apoptotic inhibitors such as venetoclax (BCL-2i) or S63845 (MCL-1i) enhanced sensitivity in a cell-specific manner. In addition, we demonstrate that both G2/M cell cycle arrest and DNA damage induction put a similar stress on DLBCL cells, thereby enhancing anti-apoptotic dependency. Therefore, genotoxic or cell cycle disrupting agents combined with specific anti-apoptotic inhibitors may be very effective in genomic unstable cancers such as DLBCL and therefore warrants further clinical evaluation.
\end{abstract}

Keywords: diffuse large B-cell lymphoma; BH3 profiling; WEE1; AZD1775; venetoclax; navitoclax

\section{Introduction}

Diffuse large B-cell lymphoma (DLBCL) is a genomically unstable cancer with multiple low-frequency mutations, somatic copy number alterations, and chromosomal translocations [1] To survive in such a genetically hazardous setting, DLBCL cells rely on cell cycle checkpoints, DNA repair, and anti-apoptotic proteins [2,3]. We have recently demonstrated that the cell cycle regulator WEE1 is highly expressed in DLBCL and is a relevant target for therapy [4]. WEE1 acts through phosphorylation of cyclin-dependent kinase 1 (CDK1/CDC2), which blocks the cell cycle at G2/M to allow time for DNA damage surveillance [5]. Under normal circumstances, factors such as cell cycle disruption and DNA damage result in induction of (intrinsic) apoptosis [6-10]. Indeed, blocking of WEE1 activity with AZD1775 results in apoptosis in solid cancers [11], lymphoma [4], and leukaemia $[12,13]$. In order to survive intrinsic apoptosis, DLBCL and many other cancers induce upregulation of various anti-apoptotic proteins, leading to apoptosis resistance, resulting in a cellular reliance on anti-apoptotic proteins commonly termed 'anti-apoptotic dependency' $[14,15]$. 
Since AZD1775 is very effectively able to induce intrinsic apoptosis, we aimed to investigate whether AZD1775 could alter the anti-apoptotic dependency and enhance the sensitivity of DLBCL cells to anti-apoptotic inhibitors (a.k.a., BH3 mimetic drugs).

The intrinsic apoptotic pathway is regulated by a complex interaction of pro- and anti-apoptotic proteins at the surface of mitochondria. Cells undergo apoptosis when this protein complex is balanced towards pro-apoptotic proteins (e.g., BIM (pro-apoptotic effector), BAD, NOXA, PUMA, BMF, HRK (pro-apoptotic sensitizers)). On the other hand, when apoptotic dependency shifts towards anti-apoptotic proteins (e.g., BCL-2, BCL-XL, BCL-W, and MCL-1) cells can avoid apoptosis [16]. Many studies in both cell lines and primary patients' samples have demonstrated the (clinical) potential of BH3 profiling, a technique that can identify a selective dependency on individual proor anti-apoptotic proteins in this complex network [17]. With this method, cells are exposed to $\mathrm{BH} 3$ peptides which enter the mitochondria and interact with anti-apoptotic proteins, resulting in a mitochondrial outer membrane permeabilization (MOMP) which can be measured using the JC-1 dye or as cytochrome-c release. Dynamic BH3 profiling (dBH3) is an ex vivo, clinically applicable variant of $\mathrm{BH} 3$ profiling which measures early changes in the apoptotic signalling cascade at the mitochondrial level, whether induced in cancer cells by treatment with classical chemotherapy or by specific anti-cancer agents [18-20].

In the present study we showed that WEE1 inhibitor AZD1775 induces cell death through apoptosis. In addition, we showed that AZD1775 significantly enhanced the dependency on anti-apoptotic proteins and enhanced sensitivity to BH3 mimetic drugs in DLBCL cells in a cell-specific manner. Similar changes in the anti-apoptotic dependency were induced by DNA damage and cell cycle arrest independently. Based on these results, we predict that AZD1775 and other similar drugs are very suitable to combine with $\mathrm{BH} 3$ mimetic drugs for the treatment of cancer.

\section{Results}

\subsection{AZD1775 Induces Apoptosis and Enhances Anti-Apoptotic Dependency in DLBCL}

DLBCL is a genomic unstable and heterogeneous cancer that shows a variety of molecular phenotypes. We previously demonstrated high expression of WEE1 in DLBCL cells compared to normal B-cells [4] and found this difference is significantly higher in DLBCL compared to other cancers (Figure S1). These data suggest that genomically unstable tumours such as DLBCL might be particularly sensitive to WEE1 inhibition. Thereto, we selected a panel of DLBCL cell lines with different genetic aberrations (Table S1). Treatment of DLBCL cell lines with WEE1 inhibitor AZD71775 induced a rapid decline in cell viability, with IC50 values ranging from 0.4 to $1.8 \mu \mathrm{M}$ (Figure $1 \mathrm{~A}$ and Table S1).

Cell death was induced by apoptosis, as measured by flow cytometry for annexin V/PI staining (Figure 1B). Apoptosis induced by AZD1775 could be observed in both a time and dose-dependent manner and could be rescued by pan-caspase inhibitor QVD (Figure S2). Next, we used dynamic BH3 profiling (Figure 1C and Table S2), which measures the changes in anti-apoptotic dependency upon treatment with AZD1775. As a result of AZD1775 treatment, cell lines OCI-LY3, SUHDL-6, SUDHL-10, and SC-1 had increased mitochondrial response to the pro-apoptotic peptide BIM at $27 \%(p=0.0058)$, $10 \%(\mathrm{~ns}), 14 \%(p=0.0480)$, and $8 \%(\mathrm{~ns})$, respectively, indicating cells were more primed to undergo apoptosis. $\mathrm{BH} 3$ profiling with a mean $\triangle \mathrm{MOMP} \geq 20 \%$ was classified as biologically relevant, even if they were not statically significant, as they often lead to significant enhanced sensitivity to BH3 mimetic drugs, indicating biological relevance. In addition, the changes induced by AZD1775 treatment could be induced in a dose-dependent manner (Figure S3A) and were significantly correlated to the percentage of apoptotic cells (Figure S3B,C). To investigate whether AZD1775-treated cells try to resist apoptosis, we next studied the dynamics of anti-apoptotic proteins MCL-1, BCL-XL, and BXL-2 in response to WEE1 inhibition as measured by mitochondrial response for NOXA, HRK, and BAD, respectively (Figure 1C). A significantly increased mitochondrial response to NOXA was observed in SUDHL-5 $(12 \%, p=0.0256)$ and in SUDHL-10 $(13 \%, p=0.0393)$, indicating increased dependency on MCL-1 
upon AZD1775 treatment. For cell lines OCI-LY3 and SC-1, a significantly increased response was observed for HRK ( $21 \% ; p=0.0866$ and $27 \% ; p=0.0223$, respectively), demonstrating WEE1 inhibition increased dependency on BCL-XL. Most cell lines showed an increased mitochondrial response to BAD upon AZD1775 treatment, which reached 43\% in OCI-LY3 ( $p=0.0195), 20 \%$ in U-2932 (ns), 23\% in SUDHL-4 $(p=0.0317), 11 \%$ in SUDHL-6 $(p=0.0223)$, and $30 \%$ in SC-1 $(p=0.0540)$. These results suggest that AZD1775 treatment leads to an increased dependency on BCL-2/BCL-XL/BCL-W. Only in cell line SUDHL-2, AZD1775 treatment did not induce changes in the anti-apoptotic dependency (Figure 1C), despite being relatively sensitive to AZD1775 treatment (Figure 1A). These results suggest that perhaps other apoptosis pathways, such as the extrinsic pathway, might be involved in the induction of apoptosis in SUDHL-2. Dynamic changes induced by AZD1775 in the different DLBCL cell lines showed no significant differences dependent on the GCB or ABC-subtype of DLBCL cell lines nor the TP53 status (Table S1). In conclusion, AZD1775 induces cell death through apoptosis, which enhanced the dependency on anti-apoptotic proteins.

A
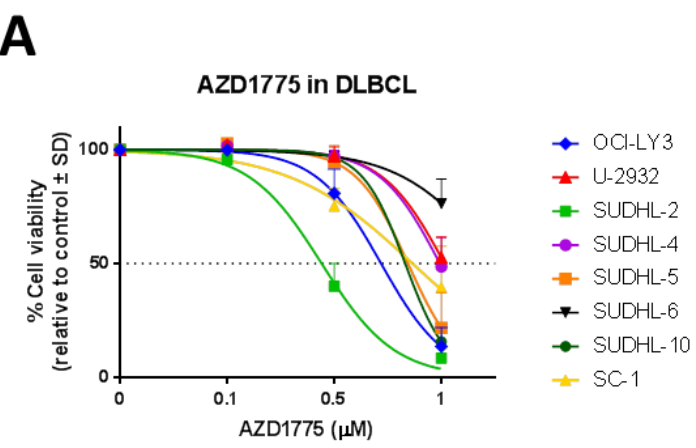

C

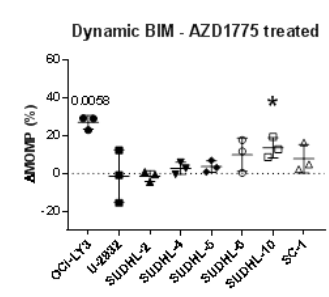

B

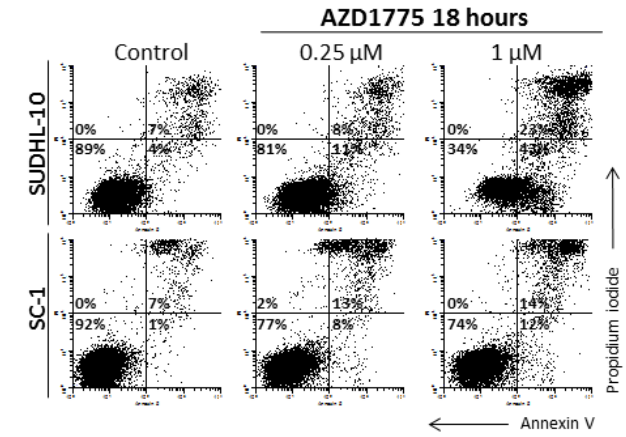

Figure 1. Apoptosis induced by AZD1775 in diffuse large B-cell lymphoma (DLBCL). (A) Cell viability analysis was measured with propidium iodide flow cytometry in DLBCL cell lines treated with AZD1775 for $72 \mathrm{~h}$. Data were plotted as the mean $\pm \mathrm{SD}(n=3)$. (B) Representative flow cytometry of apoptosis (Annexin V/Propidium iodide) induced by treatment of AZD1775 for $18 \mathrm{~h}$ in representative cell lines SUDHL-10 and SC-1. (C) Mitochondrial response of DLBCL cell lines treated with AZD1775 for $18 \mathrm{~h}$, plotted as the delta mitochondrial outer membrane permeabilization $(\triangle \mathrm{MOMP} \%) . \triangle \mathrm{MOMP} \%$ was calculated by subtracting the percentage treated MOMP from percentage untreated MOMP. Cell line SUDHL-10 was treated with $0.25 \mu$ M AZD1775, cell lines OCI-LY3, U-2932, SUDHL-2, and SUDHL-5 were treated with $0.5 \mu \mathrm{M}$ AZD1775 and cell lines SUDHL-4, SUDHL-6, and SC-1 were treated with 1 $\mu \mathrm{M}$ AZD1775. Data were plotted as the mean $\pm \mathrm{SD}(n=3)$. Statistical analysis was performed using a one-sample $t$-test as compared to untreated control cells $(* p \leq 0.05)$.

\subsection{DNA Damage and Premature Mitotic Entry Induced by AZD1775 Enhance Anti-Apoptotic Dependency}

WEE1 inhibition by AZD1775 induces both premature mitotic entry [21,22] and DNA damage [23] in various types of cancer cells. To confirm that AZD1775 has a similar effect on DLBCL cells, we analysed cell cycle distribution and $\gamma \mathrm{H} 2 \mathrm{AX}$ expression as a marker for DNA damage in the representative MCL-1 dependent cell line SUDHL-10 and the BCL-2 dependent cell line SC-1 (Figure 2A). AZD1775 treatment resulted in a dose-dependent increase in the percentage of $\mathrm{G} 2 / \mathrm{M}$ cells, indicating premature 
mitotic entry and/or prolonged mitotic arrest and a dose-dependent increase of DNA damage $(\gamma \mathrm{H} 2 \mathrm{AX}$ positive cells) in both SUDHL-10 and SC-1 (Figure 2A).

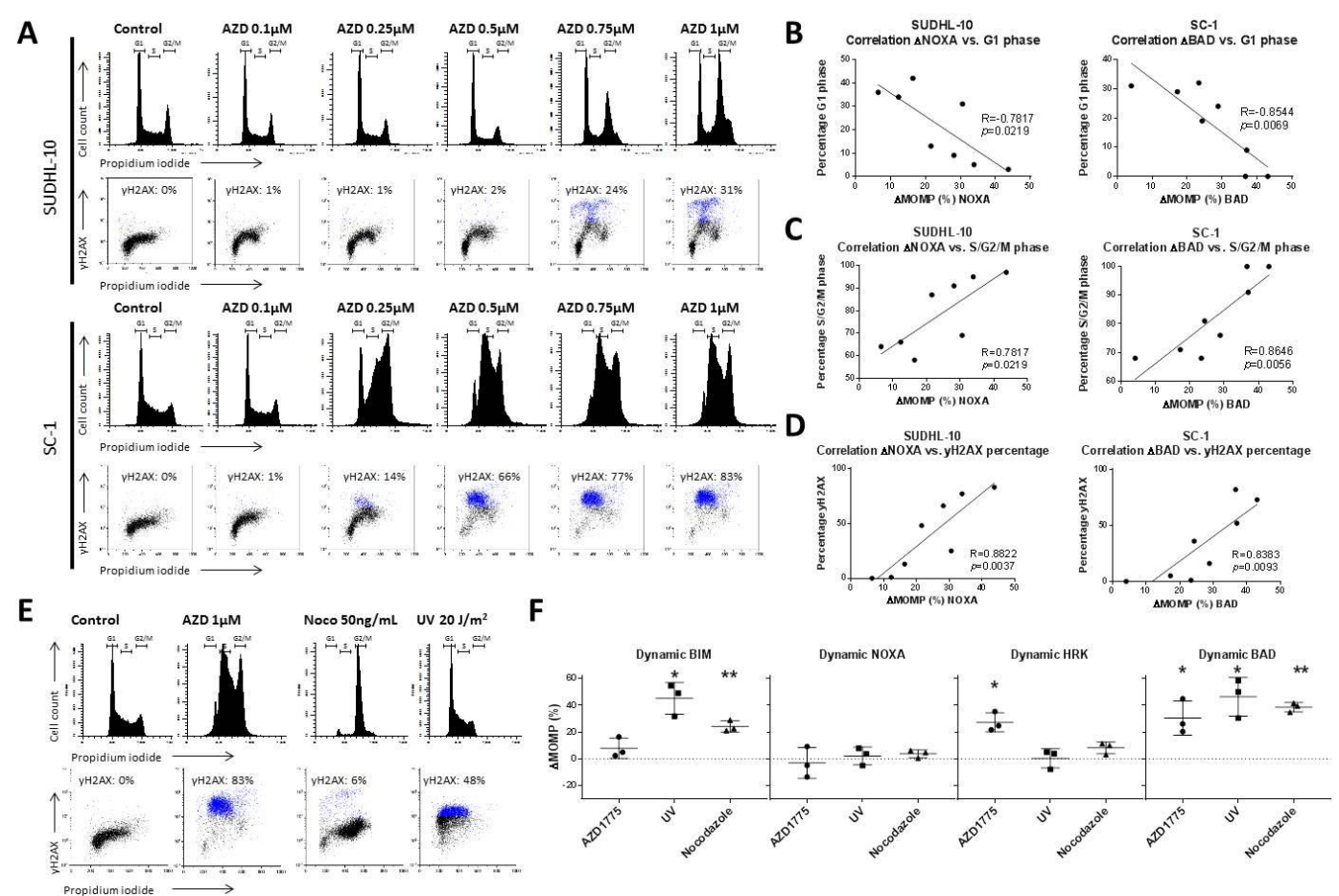

Figure 2. Cellular effect of AZD1775 treatment in DLBCL. (A) Representative examples of flow cytometry cell cycle and $\gamma \mathrm{H} 2 \mathrm{AX}$ analysis of AZD1775 in SUDHL-10 and SC-1 treated for $18 \mathrm{~h}$. (B) Correlation of the mitochondrial response ( $\triangle \mathrm{MOMP} \%$ ) for $10 \mu \mathrm{M}$ NOXA in SUDHL-10 or $0.1 \mu \mathrm{M}$ BAD in SC-1 versus the percentage of G1 phase cells treated with AZD1775. (C) Correlation of the mitochondrial response ( $\triangle \mathrm{MOMP} \%$ ) for $10 \mu \mathrm{M}$ NOXA in SUDHL-10 or $0.1 \mu \mathrm{M}$ BAD in SC-1 versus the percentage of S/G2/M phase cells treated with AZD1775. (D) Correlation of the mitochondrial response $(\triangle \mathrm{MOMP} \%$ ) of $10 \mu \mathrm{M}$ NOXA in SUDHL-10 or $0.1 \mu \mathrm{M}$ BAD in SC- 1 versus the percentage of $\gamma \mathrm{H} 2 \mathrm{AX}$ cells treated with AZD1775. Cells were treated with $0,0.1,0.2,0.3,0.4,0.5,0.75$, or $1.0 \mu \mathrm{M}$ AZD1775 for $18 \mathrm{~h}$. (E) Representative examples of cell cycle flow cytometry and $\gamma \mathrm{H} 2 \mathrm{AX}$ analysis in SC-1 treated with $1 \mu \mathrm{M}$ AZD1775, $50 \mathrm{ng} / \mathrm{mL}$ nocodazole, and $20 \mathrm{~J} / \mathrm{m}^{2} \mathrm{UV}$ radiation. (F) Dynamic BH3 profile for 0.3 $\mu \mathrm{M}$ BIM, $10 \mu \mathrm{M}$ NOXA, $10 \mu \mathrm{M}$ HRK, and $0.1 \mu \mathrm{M}$ BAD of SC-1 cells treated with AZD1775, nocodazole, and (ultra violet) UV radiation. Data were plotted as the mean $\pm \mathrm{SD}(n=3)$. Statistical analysis was performed using a one-sample $t$-test as compared to untreated control cells $\left({ }^{*} p \leq 0.05\right)$.

In addition, dynamic $\mathrm{BH} 3$ profiling showed a dose-dependent correlation between mitochondrial response to BAD in SC-1 cells treated with AZD1775 and the decrease in the percentage of G1 phase cells $(\mathrm{R}=-0.8544, p=0.0069)$ (Figure $2 \mathrm{~B})$, an increase in the percentage of $\mathrm{S} / \mathrm{G} 2 / \mathrm{M}$ phase cells $(R=0.8646$, $p=0.0056)$ (Figure 2C), and an increase in $\gamma \mathrm{H} 2 \mathrm{AX}$ levels $(R=0.8383, p=0.0093)$ (Figure 2D). Similar correlation for response to NOXA and G-1 phase cells $(R=-0.7817, p=0.0219)$, percentage of S/G2/M phase cells $(R=0.9036, p=0.0021)$, and $\gamma \mathrm{H} 2 \mathrm{AX}(R=0.8822, p=0.0037)$ were observed in SUDHL-10. These results indicate a potential relation between G2/M cell cycle arrest and increased DNA damage that could affect the anti-apoptotic dependency in DLBCL cells.

Next, we investigated if cell cycle distribution and/or DNA damage could induce changes in anti-apoptotic dependency, by exploring the ability of nocodazole to induce cell cycle arrest and of ultraviolet radiation (UV) to induce $\gamma \mathrm{H} 2 \mathrm{AX}$ (Figure 2E,F and Figure S4) in SC-1 cells. Nocodazole treatment induced an increase in the percentage of cells in the G2/M phase and a moderate $(6 \%)$ increase in $\gamma \mathrm{H} 2 \mathrm{AX}$-positive cells. UV exposure had a limited effect on the cell cycle distribution, but induced $\gamma \mathrm{H} 2 \mathrm{AX}$ in about $60 \%$ of the cells (Figure 2E and Figure S4). Dynamic BH3 profiling revealed that both 
nocodazole and UV exposure induced a significant increase in the mitochondrial response to BIM, of $24 \%(p=0.0249)$ and $45 \%(p=0.0224)$, respectively (Figure $2 \mathrm{~F})$. Similarly, response levels to BAD significantly increased, at 39\% ( $p=0.0027)$ and $46 \%(p=0.0307)$, respectively.

To further test the hypothesis that WEE1 inhibition induces changes in anti-apoptotic dependency as a result of cell cycle changes and DNA damage, we tested the combination of AZD1775 together with the CDK1 inhibitor RO3306, CDK4/6 inhibitor palbociclib, and CDK1/2 inhibitor roscovitine (Figure 3 and Figure S4), which has previously been shown to rescue AZD1775-induced DNA damage [24,25].

\section{A}

Control

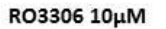

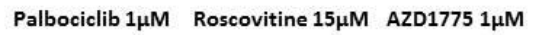
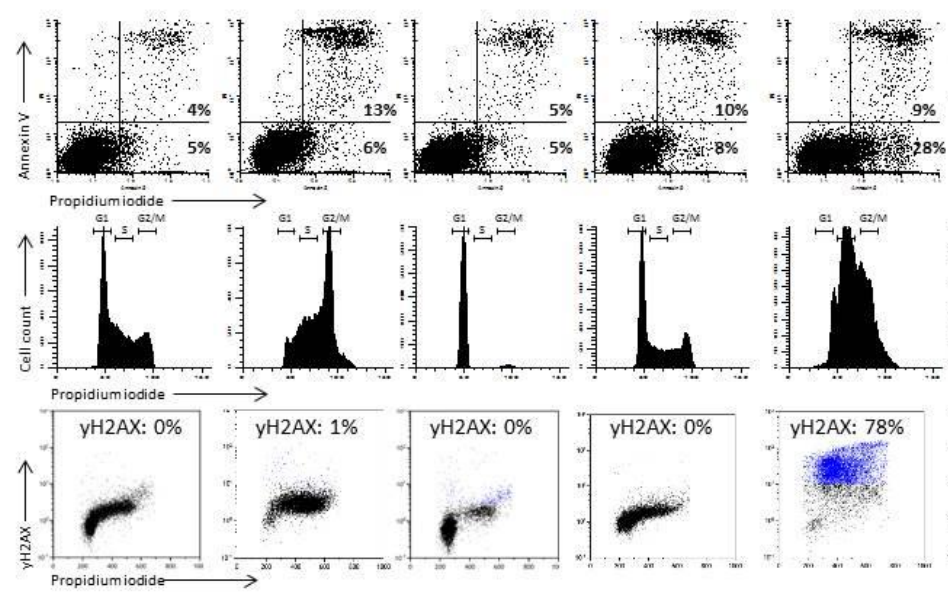

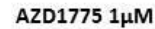

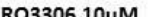

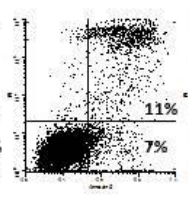
Palbociclib $1 \mu \mathrm{M}$ Roscovitine $15 \mu \mathrm{M}$

B
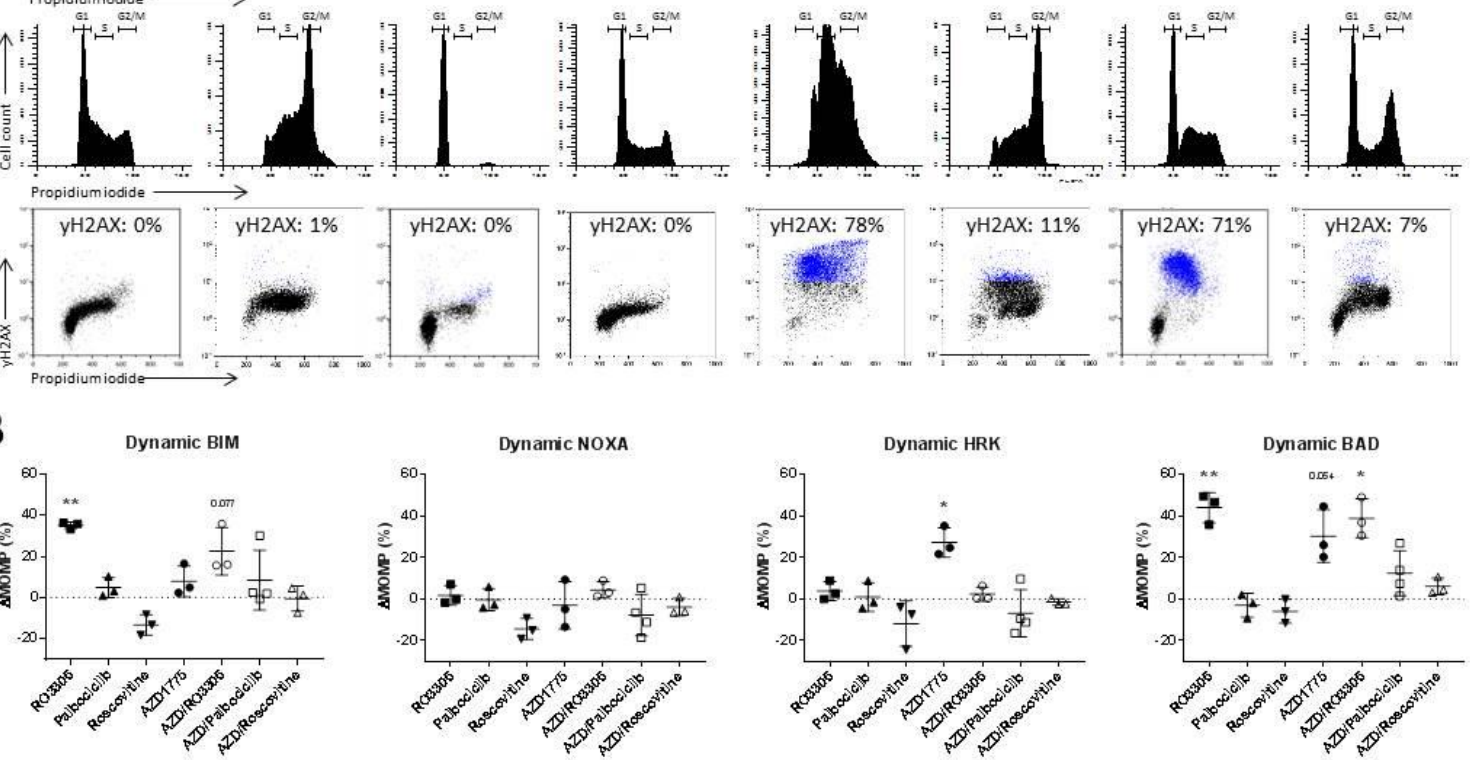

Figure 3. Rescue of AZD1775-induced cellular effects by CDK inhibitors RO3306, palbociclib and roscovitine. (A) Representative examples of apoptotic cells, cell cycle, and $\gamma \mathrm{H} 2 \mathrm{AX}$ flow cytometry analysis of SC-1 treated with $10 \mu \mathrm{M}$ RO3306, $1 \mu \mathrm{M}$ palbociclib, $15 \mu \mathrm{M}$ roscovitine, and $1 \mu \mathrm{M}$ AZD1775 after $18 \mathrm{~h}$ of incubation. (B) Dynamic BH3 profile for $0.3 \mu \mathrm{M}$ BIM, $10 \mu \mathrm{M}$ NOXA, $10 \mu \mathrm{M}$ HRK, and $0.1 \mu \mathrm{M}$ BAD of SC-1 cells treated with RO3306, palbociclib, roscovitine, and AZD1775 after $18 \mathrm{~h}$ of incubation. Delta mitochondrial outer membrane permeabilization $(\triangle \mathrm{MOMP} \%)$ was calculated by subtracting the percentage treated MOMP from percentage untreated MOMP. Data were plotted as the mean $\pm \mathrm{SD}(n=3)$. Statistical analysis was performed using a one-sample $t$-test as compared to untreated control cells $\left({ }^{*} p \leq 0.05\right)$.

Treatment of SC-1 cells with the (single agent) roscovitine had little effects on either apoptosis levels, the cell cycle, or levels of DNA damage (Figure 3A,B). However, when combined with AZD1775, roscovitine significantly reduced DNA damage levels (from $75 \%$ to $7 \%, p=0.0024$ ) and largely prevented alterations in cell cycle distribution (Figure 3A,B). Treatment with roscovitine alone or in combination with AZD1775 did not lead to an altered dynamic BH3 profile, demonstrating that a full rescue of both cell cycle distribution and DNA damage prevents anti-apoptotic changes induced by AZD1775 (Figure 3B). In addition, we tested CDK1 inhibitor RO3306 (Figure 3A,B and Figure S5), which rescues only AZD1775-induced DNA damage, and CDK4/6 inhibitor palbociclib (Figure 3A,B and Figures S5 and S6), which rescues only AZD1775-induced cell cycle arrest. Neither partial rescue of DNA damage (Figure S4) nor cell cycle distribution (Figure 3A) were sufficient to prevent 
anti-apoptotic changes induced by AZD1775. Based on these results, we conclude that both DNA damage and cell cycle disruption induced independently changes in the mitochondria and enhanced anti-apoptotic dependency.

\subsection{AZD1775 Increases Sensitivity to BCL-2 and MCL-1 Inhibitors in DLBCL}

Now that we have established that DNA damage and cell cycle disruption by AZD1775 (Figure 2) is sufficient to induce intrinsic apoptosis (Figures S1-S3) and enhance dependency on anti-apoptotic proteins to prevent apoptosis, we next studied the combination of AZD1775 with anti-apoptotic inhibitors. We used SC-1 as a cell line with enhanced dependency on BCL-2/BCL-XL/BCL-W, and SUDHL-10 and SUDHL-5 as cell lines with enhanced dependency on MCL-1 as predicted by BH3 profiling (Figure 1C). Combined treatment of AZD1775 and venetoclax caused a 10-fold decrease in cell viability in SC-1 (Figure 4A).

A
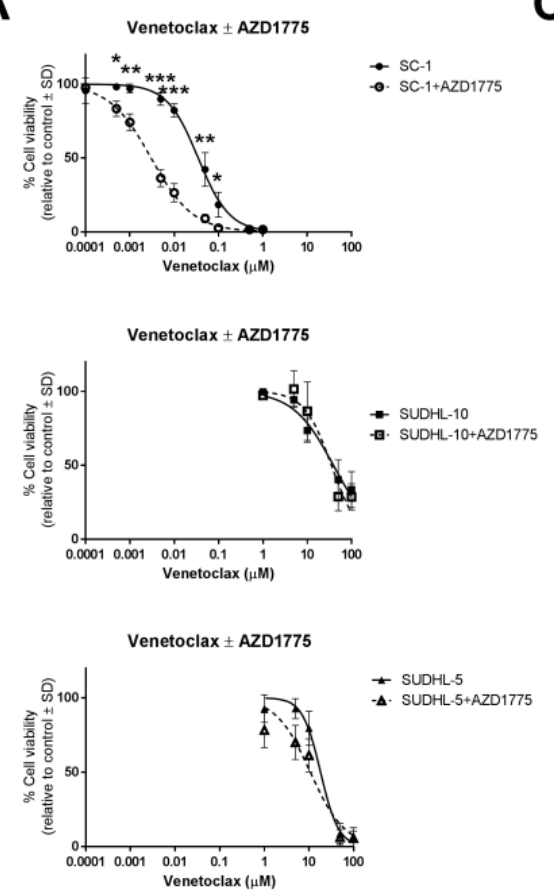

B

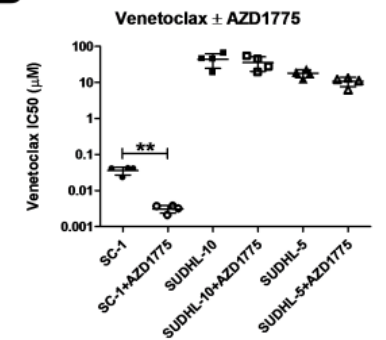

C
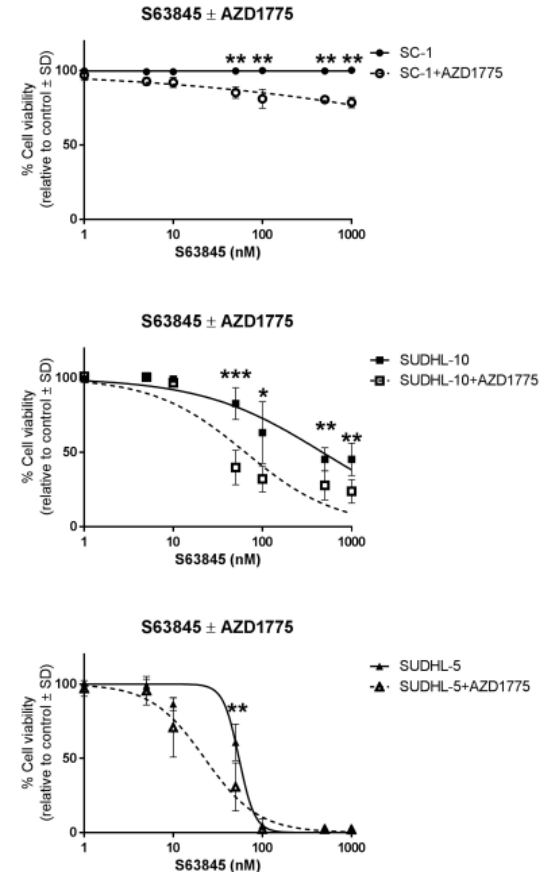

D

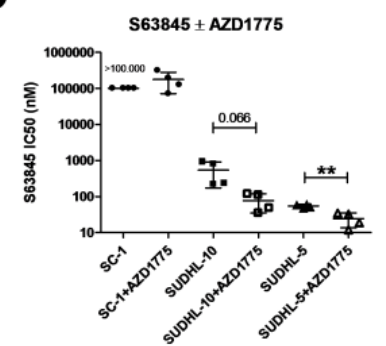

Figure 4. Combination of AZD1775 and BH3 mimetic drugs in DLBCL cell lines. (A) Cell viability flow cytometry data for the cell lines SC-1, SUDHL-5, and SUDHL-10 pre-treated with $1 \mu \mathrm{M}$ AZD1775 and incubated with increasing concentrations of venetoclax. Data were normalized to the control and plotted as the mean $\pm \mathrm{SD}(n=4)$. (B) Venetoclax IC50 values as calculated from cell viability curves. Data were plotted as the mean \pm SD $(n=4)$. (C) Cell viability flow cytometry data for SC-1, SUDHL-5, and SUDHL-10 pre-treated with $1 \mu \mathrm{M}$ AZD1775 and incubated with increasing concentrations of S63845. Data were normalized to the control and plotted as the mean $\pm \operatorname{SD}(n=4)$. (D) S63845 IC50 values as calculated from cell viability curves. Data plotted as the mean $\pm \operatorname{SD}(n=4)$. Statistical analysis was performed using a two-tailed paired $t$-test $\left({ }^{*} p \leq 0.05\right)\left({ }^{* *} p \leq 0.01\right)$. 
A significant decline in viability from $82 \%$ to $26 \%(p<0.0001)$ was observed when $0.01 \mu \mathrm{M}$ venetoclax was combined with $1 \mu \mathrm{M}$ AZD1775, resulting in an IC50 decrease from $0.04 \mu \mathrm{M}$ in AZD1775-untreated cells to $0.003 \mu \mathrm{M}$ in AZD1775-treated cells for venetoclax ( $p=0.0039$, Figure 4B). In the MCL-1 dependent cell lines SUDHL-10 and SUDHL-5, as expected, co-treatment with AZD1775 did not enhance sensitivity to venetoclax. In these cell lines, AZD1775 induced enhanced dependency on MCL-1, suggesting cells would become more sensitive to MCL-1 inhibitor S63845. Combined treatment of $1 \mu \mathrm{M}$ AZD1775 and S63845 indeed caused a dose-dependent decrease in cell viability in the cell line SUDHL-5 (from $61 \%$ to $31 \%(p=0.0024)$ at $50 \mathrm{nM} \mathrm{S63845)}$ ) and in cell line and SUDHL-10 (from $83 \%$ to $40 \%(p=0.0003)$ at $50 \mathrm{nM} \mathrm{S63845)} \mathrm{(Figure} 4 \mathrm{C}$ ). These changes resulted in a 7-fold decrease in IC50 values for S63845 in SUDHL-10 (from 544 to $77 \mathrm{nM}, p=0.0658$ ) and a 2-fold decrease in IC50 values for S63845 in SUDHL-5 (from 61 to $35 \mathrm{nM}, p=0.0028$ ) (Figure 4D). No effect on cell viability was observed when AZD1775 was combined with S63845 in SC-1 (Figure 1C). These results confirm that the changes observed in dynamic BH3 profiling upon AZD1775 treatment enhance dependency on anti-apoptotic proteins, a dependency that can be effectively exploited through targeting with the appropriate $\mathrm{BH} 3$ mimetic.

\subsection{AZD1775 Alters the Anti-Apoptotic Dependency of Patient-Derived DLBCL Cells}

Finally, we studied anti-apoptotic dependency and the dynamic BH3 profile following AZD1175 treatment of lymphoma cells from a DLBCL patient (Figure S7). Efficient apoptotic priming was observed (Figure 5A), and dynamic BH3 profiling (Figure 5B) showed an increase in the mitochondrial response to $\mathrm{BAD}(56 \%$ at $0.1 \mu \mathrm{M} \mathrm{BAD})$ but not to HRK after AZD1775 treatment, suggesting an increased sensitivity to BCL-2 or BCL-W inhibitors, but not to BCL-XL inhibitors.

A

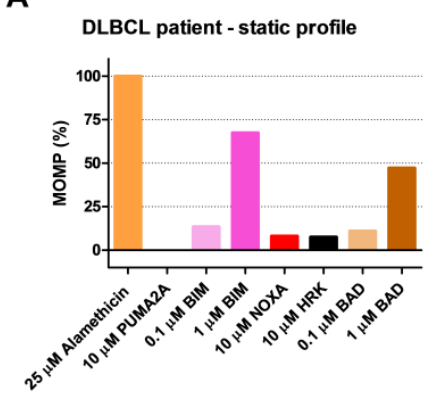

C

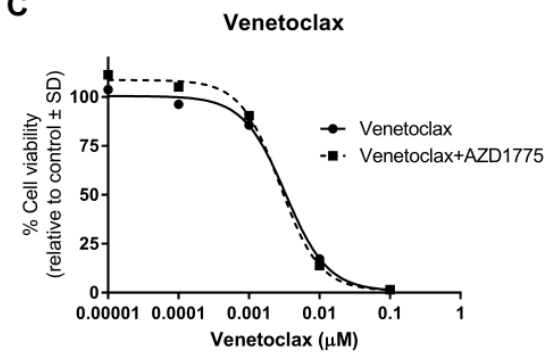

B

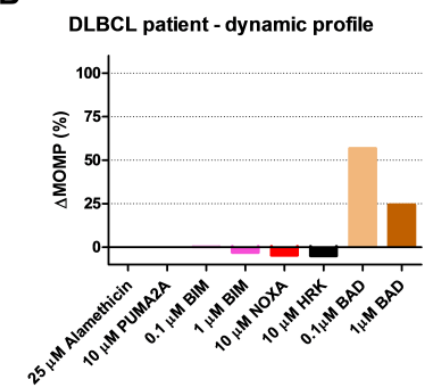

D

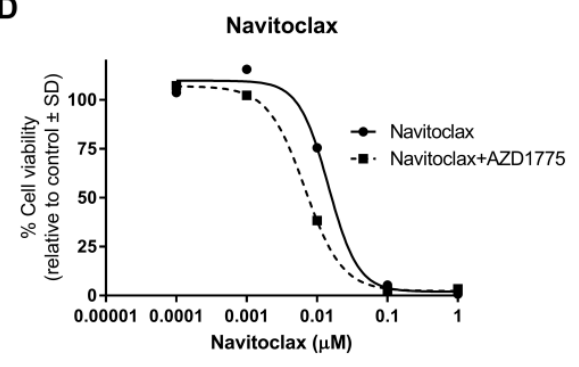

Figure 5. Dynamic BH3 profile and validation experiments in a DLBCL patient treated with AZD1775. (A) Static BH3 profile and (B) dynamic BH3 profile of DLBCL patient cells treated with $0.5 \mu \mathrm{M}$ AZD1775 for $18 \mathrm{~h}$. Alamethicin and PUMA2A were used as positive and negative controls, respectively. Delta mitochondrial outer membrane permeabilization $(\triangle \mathrm{MOMP} \%)$ was calculated by subtracting the percentage treated MOMP from percentage untreated MOMP. (C) Cell viability flow cytometry data for DLBCL patient cells pre-treated with AZD1775 for $18 \mathrm{~h}$ and incubated with increasing concentrations of venetoclax and (D) navitoclax for $24 \mathrm{~h}$. Data were normalized to the control.

These findings were validated by ex vivo treatment of lymphoma cells with AZD1775 at increasing concentrations of venetoclax (BCL-2i) or navitoclax (BCl-2i, BCl-Xli, and BCL-Wi). Venetoclax did not 
alter cell viability (Figure 5C), but navitoclax induced a 2-fold decrease in cell viability (Figure 5D), together with a decrease in IC50 from $0.015 \mu \mathrm{M}$ for navitoclax alone to $0.0067 \mu \mathrm{M}$ for navitoclax combined with AZD1775, indicating a dependency on BCL-W. We were unable to study the effect of BCL-W inhibition, as specific BCL-W inhibitors are not currently available. Taken together, these data emphasize the potential of AZD1775 combined with BH3 mimetics in the treatment of DLBCL patients and underscore the clinical utility of $\mathrm{BH} 3$ profiling.

\section{Discussion}

In genomically unstable cancers such as DLBCL, WEE1 is highly expressed and a relevant target for therapy. Since the WEE1 inhibitor AZD1775 is indeed effective in inducing apoptosis we investigated if it could alter anti-apoptotic dependency and increase sensitivity to BH3 mimetic drugs in DLBCL cell lines and patient material. Our findings demonstrate that (1) AZD1775 induces cell death through apoptosis; (2) AZD1775-mediated inhibition of WEE1 alters the anti-apoptotic dependency in DLBCL; (3) combination of AZD1775 with cell-specific anti-apoptotic inhibitors (such as venetoclax) leads to enhanced potency; (4) both DNA damage and G2/M arrest induced by WEE1 inhibition independently enhances dependency on anti-apoptotic proteins. Based on these results, we propose a model in which cell cycle disruption (such as premature mitotic entry or G2/M arrest) and DNA damage can induce changes in the mitochondrial response, resulting in an altered dependency on anti-apoptotic proteins in DLBCL cells (Figure 6).

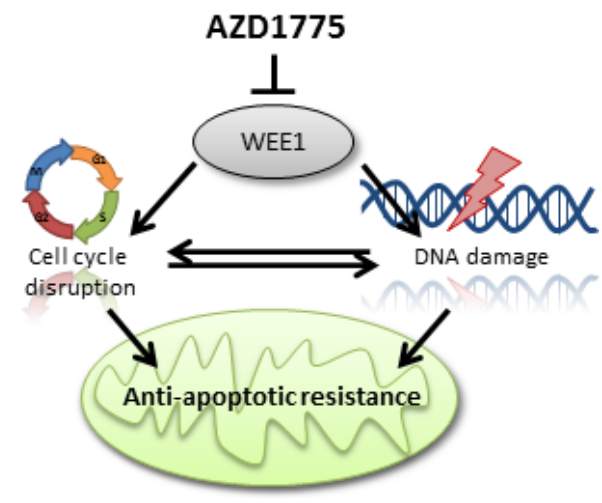

Figure 6. Proposed mechanism for the relation between inhibition of cell cycle regulator WEE1 and the resistance of apoptosis. As a result of WEE1 inhibition cells are able to prematurely enter mitosis, without proper repair of genomic aberrations or mutations. This elimination of the checkpoint at the transition from the G2-phase into the M-phase leads to continuous cell cycling and results in high levels of DNA damage that activate apoptosis pathways. To prevent apoptosis, cancerous cells become increasingly dependent on anti-apoptotic proteins such as BCL-2, MCL-1, BCL-XL, and BCL-W, which ensure survival under high levels of genomic stress.

In normal B-cell development, dependency on anti-apoptotic proteins changes during B-cell maturation. Naïve B-cells are dependent on BCL-2, GC B-cells shift to dependency on MCL-1, memory B-cells are once more dependent on BCL-2, while plasma cells depend on BCL-XL [26]. The $t(14 ; 18)$ IGH-BCL-2 translocation, found in $\sim 20 \%$ of DLBCL patients, is a hallmark of follicular lymphoma and is more commonly found in the germinal centre derived-DLBCL subtype [14,27]. BCL-W has a potentially important role in B-cell survival, as overexpression of the BCL-W gene is associated with a worse prognosis in specific DLBCL cases [28]. Using baseline BH3 profiles and sensitivity to BH3 mimetic drugs, we showed that DLBCL cell lines are dependent on multiple anti-apoptotic proteins. These associations showed no direct relationship to the cell of origin, thus highlighting the heterogeneity of DLBCL.

To date, the primary application of dynamic $\mathrm{BH} 3$ profiling has been measurement of responses to chemotherapy [17]. Here, we were able to accurately predict changes in anti-apoptotic dependency 
profiles as a result of WEE1 inhibition by AZD1775. These dependencies changed both quantitatively and qualitatively. For example, dynamic $\mathrm{BH} 3$ profiling highlighted interesting shifts in dependency, such as the shift to dependency on BCL-XL after AZD1775 treatment of the previously BCL-XL independent SC1 cell line. This shows that tumour cells can shift their dependency from one anti-apoptotic protein to another. Unfortunately, current multiple anti-apoptotic protein inhibitors, including those targeting BCL-XL such as navitoclax, are poorly tolerated and have significant side effects [29]. Inhibition of BCL-XL also shortens platelet lifespan [30], hampering use of these agents. Nevertheless, as tumour cells appear able to use and adapt their dependency to multiple anti-apoptotic proteins, there is clearly a need for new, tolerated multi-target inhibitors in order to prevent resistance and relapse in DLBCL patients. Our results demonstrate the broad range of effects of AZD1775 on cellular states, including the ability to influence apoptotic pathways, clearly highlighting potential therapeutic targets. It can be argued that analyses of protein levels by western blot or immunohistochemistry, perhaps including RNA expression levels, are suboptimal approaches when determining the anti-apoptotic dependency of tumour cells. By contrast, static BH3 profiling of cell lines or primary patient samples has proven itself as a fast and reliable tool to establish the functionality of and dependency on anti-apoptotic proteins.

Treatment of solid cancers [11] and leukaemia [12,13] with inhibitors of the cell cycle regulator WEE1 has proven successful in early clinical trials, as WEE1 inhibitors result in cell cycle disruption [21,22], induction of DNA damage [23,31] and eventually, induction of apoptosis. Our data highlighted a novel effect of WEE1 inhibition, an altered dependency on anti-apoptotic proteins. Rescue experiments preventing either DNA damage and/or G2/M cell cycle arrest after WEE1 inhibition showed that both events are able to induce changes in anti-apoptotic dependency, either individually or in combination. Mitochondrial fission at mitosis is known to be tightly regulated by the CDK1-cyclinB complex, which phosphorylates the GTPase Drp1 and thus induces mitochondrial fragmentation to promote mitochondrial fission [32,33]. Loss of Drp1 results in dysfunctional mitochondrial fission, persistent mitochondrial hyperfusion, delayed G2/M cell cycle progression, replication stress, DNA damage, ATM activation, and genomic instability [34]. Since WEE1 is the main regulator of the CDK1-cyclinB complex, loss of WEE1 will induce premature mitochondrial fragmentation, destabilizing the connection between cell cycle division and mitochondrial homeostasis. Similarly, activation of the DNA damage response protein ATM in response to double-stranded DNA breaks results in phosphorylation of the BH3 activator protein BID, initiating DNA damage-induced apoptosis [35]. Here, we show a novel direct link between the cell cycle, DNA damage and mitochondrial apoptosis, in which either premature mitotic entry or DNA damage induced by WEE1 inhibition alters anti-apoptotic dependency. Although this mechanism was not tested in other cancer types, based on the fundamental role of WEE1 in cell cycle regulation we expect a similar association and response in other cell types. With the current development of BH3 mimetics, WEE1 inhibition would be an excellent candidate for dose reduction combination therapy in treatment-naïve patients, or could be applied as a $\mathrm{BH} 3$ mimetic sensitizer in WEE1 inhibitor-resistant tumours.

In conclusion, we demonstrated for the first time that WEE1 inhibition in DLBCL can lead to further sensitization to anti-apoptotic inhibitors, revealing a novel mechanism and as yet unexplored application for WEE1 inhibition. Using cell cycle and DNA damage rescue experiments, we unraveled the mechanism underlying changes in anti-apoptotic dependency, highlighting mechanisms that may have applications in the treatment of other cancers. These findings suggest important applications for WEE1 as a novel therapeutic treatment approach, but also suggest broader possibilities for genotoxic drugs, cell cycle deregulators, or DNA damage response inhibitors in combination with BH3 mimetic drugs. Finally, we showed that combining WEE1 inhibition with dynamic BH3 profiling represents an educated approach to guided therapy, and may lead to novel strategies in optimalized/personalized treatment selection in DLBCL patients. 


\section{Materials and Methods}

\subsection{Cell Lines and Culture Conditions}

The DLBCL cell lines U-2932 (ABC), SUDHL-2 (ABC), SUDHL-4 (GCB), and SC-1 (GCB) were cultured in suspension in Roswell Park Memorial Institute medium 1640 (RPMI 1640; Lonza BioWhittaker, Walkersville, MD, USA) with 10\% fetal bovine serum (FBS; HyClone Thermo Scientific, Waltham, MA, USA), 1\% Penicillin-Streptomycin (PS; Lonza BioWhittaker) and 1\% glutamine (Lonza BioWhittaker). The DLBCL cell lines OCILY3 (ABC), SUDHL-5 (GCB), SUDHL-6 (GCB), and SUDHL-10 (GCB) were cultured in suspension in RPMI 1640 with 20\% FBS, $1 \%$ PS and 1\% glutamine. All cell lines were cultured at $37^{\circ} \mathrm{C}$ with $5 \% \mathrm{CO} 2$ in a humidified atmosphere. The identity of our cell lines was checked on a regular basis. Cell line characteristics are given in Table S1.

\subsection{Patient Material and METC Statement}

Patient material was acquired in accordance with international regulations and professional guidelines (the Declaration of Helsinki and the International Conference on Harmonization Guidelines for Good Clinical Practice). Material used in this project was obtained from anonymous rest material. The medical ethics review board waives the need for approval if rest material is used, under the law in the Netherlands and waives the need for informed consent when patient anonymity is assured.

\subsection{BH3 Profiling—Plate-Based Assay}

Cells were incubated at $0.5 \times 10^{6}$ cells/mL for $18 \mathrm{~h}$ with AZD1775 (WEE1 inhibitor, Selleckchem No.S1525, Houston, TX, USA) (Figure S8), palbociclib (CDK4/6 inhibitor, Selleckchem No.S1116), RO3306 (CDK1 inhibitor, Selleckchem No.S7747), roscovitine (CDK2 inhibitor, Selleckchem No. S1153), nocodazole (M1404, Sigma Aldrich, St. Louis, MO, USA), or ultraviolet (UV) radiation (IBL 637, CisBioInternational, Gif-sur-Yvette, France). After incubation, cells were washed with mannitol experimental buffer (MEB) (150 mM D-mannitol (M9647, Sigma Aldrich,), 10 mM HEPES (H3375, Sigma Aldrich), $50 \mathrm{mM} \mathrm{KCl}$ (1.04936, Merck, Darmstadt, Germany), $20 \mathrm{nM} \mathrm{EGTA} \mathrm{(E4378,} \mathrm{Sigma} \mathrm{Aldrich),} 20 \mathrm{nM}$ EDTA (11280, Serva Electrophoresis, Heidelberg, Germany), 0.1\% BSA (11930, Serva Electrophoresis), $5 \mathrm{mM}$ succinate (1.00682, Merck) in $\mathrm{dH} 2 \mathrm{O}, \mathrm{pH} 7.5$ ) and resuspended at $3.2 \times 10^{6}$ cells $/ \mathrm{mL}$ in MEB. A cell suspension was mixed 1:1 with $4 \mu \mathrm{M}$ JC-1 permeabilization/staining solution ((ENZ-52304, Enzo Life Sciences, Farmingdale, NY, USA), 0.004\% digitonin (1500 643, Boehringer Mannheim, Mannheim, Germany), $20 \mathrm{mM} \beta$-mercaptoethanol (8.05740, Merck) and $40 \mu \mathrm{g} / \mathrm{mL}$ oligomycin (O4876, Sigma Aldrich) prepared in MEB) and incubated at room temperature in the dark for $10 \mathrm{~min}$. BIM, PUMA, BAD, NOXA, MS1, HRK, BMF, and PUMA2A (JPT Peptide Technologies, Berlin, Germany) were prepared in MEB in a black flat-bottom non-treated polystyrene 96-well plate (3915 Costar, Corning Incorporated, Kennebunk, ME, USA). Peptide sequences used for the assay were identical to those described in Ryan and Letai [18]. Plates were either used directly or sealed (Silverseal sealer ref 676090, Greiner-Bio-One, Frickenhausen, Germany), frozen at $-80^{\circ} \mathrm{C}$ and thawed for $1 \mathrm{~h}$ at room temperature before use. Cells in permeabilization/staining solution were added to the plate 1:1 at a final volume of $100 \mathrm{uL}$ and shaken for $15 \mathrm{~s}$, followed by measurement of fluorescence (excitation $545 \mathrm{~nm}$, emission $590 \mathrm{~nm}$ ) every $5 \mathrm{~min}$ for $2 \mathrm{~h}$ at $30^{\circ} \mathrm{C}$ (Varioskan). All experiments were performed in triplicates or quadruplicate. The area under the curve (AUC) was calculated as a percentage of mitochondrial outer membrane permeabilization (MOMP) and normalized to PUMA2A (negative control) and FCCP (positive control) with the formula: 1 - ((AUC sample - AUC FCCP) $\div$ (AUC PUMA2A - AUC FCCP) $\times 100 \%$. The dynamic $\mathrm{BH} 3$ profile $(\triangle \mathrm{MOMP})$ was calculated by subtracting the percentage treated MOMP from percentage untreated MOMP. Based on the specific interacting partners of the BH3 peptides [18], the cell specific anti-apoptotic dependency of cells can be established. BH3 profiling with a mean $\triangle \mathrm{MOMP} \geq 20 \%$ were classified as biologically relevant, even if they were not always statically significant. $\triangle \mathrm{MOMP}$ changes for the BIM peptide indicates cells have become more primed for apoptosis or closer to their apoptotic threshold. Similarly, $\triangle$ MOMP changes for NOXA, HRK, 
or BAD indicate cells have become more dependent on MCL-1, BCL-XL, or BCL-2/XL/W, respectively. Together, these data can predict on which anti-apoptotic protein cells are dependent, and how this changes upon inhibitor treatment.

\subsection{BH3 Profiling-Flow Cytometry-Based Assay}

Peripheral blood mononuclear cells were isolated from a DLBCL patient, washed with $10 \%$ FBS RPMI, incubated at $1.0 \times 10^{6}$ cells/mL for $18 \mathrm{~h}$ with $1 \mu \mathrm{M}$ AZD1775 (WEE1 inhibitor, Selleckchem) in RPMI medium supplemented with 10\% fetal bovine serum, 1\% Penicillin-Streptomycin, and 1\% glutamine. After incubation, cells were washed with 1\% BSA (11930, Serva) in PBS and incubated with CD19-APC antibody (IQP-106A, IQ Products, Groningen, The Netherlands) for $30 \mathrm{~min}$ at $4{ }^{\circ} \mathrm{C}$. After staining, cells were washed with and suspended in MEB at 3-5 $\times 10^{6}$ cells $/ \mathrm{mL}$. Cells were incubated with peptides in $0.001 \%$ digitonin at $25^{\circ} \mathrm{C}$ for $60 \mathrm{~min}$. The reaction was stopped by addition of $2 \%$ formaldehyde (104005, Merck) in PBS and cells were fixed in 2\% formaldehyde for 20 min at room temperature. Cells were washed in 1\% BSA in PBS and stained overnight for intracellular cytochrome-c in PBS containing 1\% BSA in PBS, 0.2\% Tween 20 (P7949 Sigma Aldrich) and 1:400 Alexa Fluor 488 anti-cytochrome-c antibody (612308, BioLegend 9727 Pacific Heights Blvd. San Diego, CA 92121, USA) at $4{ }^{\circ} \mathrm{C}$. Before analysis, cells were washed with 1\% BSA in PBS and flow cytometry analysis was performed on a FACSCalibur (BD Biosciences, 1 Becton Drive, Franklin Lakes, NJ 07417-1880, USA). Data were normalized to PUMA2A (negative control) and alamethicin (positive control). The dynamic $\mathrm{BH} 3$ profile $(\triangle \mathrm{MOMP})$ was calculated by subtracting the percentage treated MOMP from percentage untreated MOMP.

\subsection{Flow Cytometry-Based Apoptosis Assay}

Cells were incubated at $0.1 \times 10^{6}$ cells/mL with AZD1775 (WEE1 inhibitor, Selleckchem) with or without QVD-Oph (pan-caspase inhibitor, Selleckchem, No. S7311) for 18, 24 or 72 h. After incubation, cells were washed with 1\% BSA in PBS and stained with Annexin V-FITC (IQP-120F, IQ products, Groningen, The Netherlands) for 20 min on ice. Cells were washed with 1\% BSA in PBS and stained with propidium iodide (Sigma Aldrich) to assess early apoptosis (Annexin V positive/propidium iodide negative) and late apoptosis (Annexin V positive/propidium iodide positive). Apoptosis was measured by flow cytometry (FACSCalibur, BD Biosciences). Data were analysed in Winlist 3D (Verity Software house, Topsham, ME, USA).

\subsection{Flow Cytometry-Cell Cycle, $\gamma H 2 A X$, and $p H 3$ with DNA Content}

For cell cycle analysis, $0.2 \times 10^{6}$ cells/mL were treated for the indicated time points, washed with 1\% BSA/PBS and resuspended in solution containing 0.1\% sodium citrate (A0158348, Merck, Kenilworth, New Jersey, USA), 0.01\% propidium iodide (P4170, Sigma Aldrich), 0.002\% RNase A (R4875, Sigma Aldrich), and 0.3\% Triton X100 (T9284, Sigma Aldrich). Samples were processed on a BD FACSCalibur 2 and analysed with ModFit LT (Verity Software House, Topsham, ME , USA).

For $\gamma \mathrm{H} 2 \mathrm{AX}$ analysis, $0.2 \times 10^{6}$ cells $/ \mathrm{ml}$ were treated for the indicated time points and then stained with mouse anti- $\gamma \mathrm{H} 2 \mathrm{AX}-\mathrm{AlexaFluor}-647$ (clone 2F3, \#613408, BioLegend) and propidium iodide solution (P4170, Sigma Aldrich) according to the protocol provided with the eBioscience ${ }^{\mathrm{TM}}$ Foxp3/Transcription Factor Staining Buffer Set (ThermoFisher, \#00-5523-00, Waltham, MA, USA). Samples were processed on a MACSQuant and the data were analyzed using Kaluza 1.5 analysis software (Beckman, Brea, CA, USA).

\subsection{Flow Cytometry-Based Viability Assay}

Cells were incubated at $0.1 \times 10^{6}$ cells/mL with AZD1775 (WEE1 inhibitor, Selleckchem) for $18 \mathrm{~h}$. After washing with 1\% BSA in PBS, cells were incubated with venetoclax/ABT-199 (BCL-2-selective inhibitor, Selleckchem), S63845 (MCL-1 inhibitor, Selleckchem), or navitoclax/ABT-263 (BCL-XL/BCL-2/BCL-W inhibitor Selleckchem) for $48 \mathrm{~h}$ at $37^{\circ} \mathrm{C}$. After incubation, cells were washed 
with 1\% BSA in PBS and stained with propidium iodide (Sigma Aldrich) to assess cell viability by flow cytometry (FACSCalibur, BD Biosciences). Data were analysed in Winlist 3D (Verity Software House, Topsham ME, USA).

\subsection{Statistical Analysis}

A one-sample $t$-test was used to assess the significance of the dynamic $\mathrm{BH} 3$ profiling results ( $n=3$ independent repeats). Comparison of IC50 values between untreated and AZD1775-treated samples was done using a two-tailed paired $t$-test. Correlation analysis was carried out using Pearson's correlation test. ${ }^{*}$ indicates $p \leq 0.05,{ }^{* *}$ indicates $p \leq 0.01$, and ${ }^{* * *}$ indicates $p \leq 0.001$.

\section{Conclusions}

In conclusion, we demonstrate that treatment of cells with WEE1 inhibitor AZD1775 can enhance the dependency on anti-apoptotic proteins and enhanced sensitivity to $\mathrm{BH} 3$ mimetic drugs. In addition, we showed that DNA damage and cell cycle arrest independently induce similar changes in the anti-apoptotic dependency. Therefore, we predict that other DNA damage-inducing of cell cycle arresting agents will synergize with $\mathrm{BH} 3$ mimetic drugs.

Supplementary Materials: The following are available online at http://www.mdpi.com/2072-6694/11/11/1743/s1. Table S1: Characteristics of DLBCL cell lines, Table S2: BH3 treatment schedule for DLBCL cell lines, Figure S1: Gene expression levels of WEE1 in multiple cancer types, Figure S2: Time and dose-dependent induction of apoptosis by AZD1775 in DLBCL, Figure S3: Representative example of the dose-response effect of AZD1775 on mitochondrial outer membrane permeabilization, Figure S4: DNA damage induced by AZD1775, nocodazole, or UV treatment, Figure S5: DNA damage induced by AZD1775 alone or combined with CDK inhibitors, Figure S6: Correlation of dynamic BAD BH3 profile and $\gamma \mathrm{H} 2 \mathrm{AX}$ after Palbociclib and AZD1775, Figure S7: DLBCL patient characteristics. Figure S8: DLBCL cells treated with AZD1775.

Author Contributions: Conceptualization, M.R.W.d.J., M.L., L.V., and T.v.M.; Data curation, M.R.W.d.J., M.L., B.R., and P.H.; Formal analysis, M.R.W.d.J.; Funding acquisition, T.v.M.; Supervision, A.v.d.B., L.V., and T.v.M.; Validation, M.R.W.d.J., M.L., B.R., and P.H.; Writing—original draft, M.R.W.d.J.; Writing—review and editing, M.R.W.d.J., M.L., B.R., M.N., G.H., A.v.d.B., E.A., L.V., and T.v.M.

Funding: This research was supported by a Bas Mulder grant from Alpe d'HuZes/Dutch Cancer Society (RUG 2014-6727) and a Mandema Stipendium (both awarded to T. van Meerten).

Acknowledgments: The authors thank the University Medical Center Groningen Flow Cytometry department for the services provided.

Conflicts of Interest: The authors declare no conflict of interest.

\section{References}

1. Bakhoum, S.F.; Danilova, O.V.; Kaur, P.; Levy, N.B.; Compton, D.A. Chromosomal instability substantiates poor prognosis in patients with diffuse large B-cell lymphoma. Clin. Cancer Res. 2011, 17, 7704-7711. [CrossRef] [PubMed]

2. Derenzini, E.; Agostinelli, C.; Imbrogno, E.; Iacobucci, I.; Casadei, B.; Brighenti, E.; Righi, S.; Fuligni, F.; Di Rora, A.C.L.; Ferrari, A.; et al. Constitutive activation of the DNA damage response pathway as a novel therapeutic target in diffuse large B-cell lymphoma. Oncotarget 2015, 6, 6553-6569. [CrossRef] [PubMed]

3. Ranuncolo, S.M.; Polo, J.M.; Dierov, J.; Singer, M.; Kuo, T.; Greally, J.; Green, R.; Carroll, M.; Melnick, A. Bcl-6 mediates the germinal center $\mathrm{B}$ cell phenotype and lymphomagenesis through transcriptional repression of the DNA-damage sensor ATR. Nat. Immunol. 2007, 8, 705-714. [CrossRef] [PubMed]

4. $\quad$ de Jong, M.R.W.; Visser, L.; Huls, G.; Diepstra, A.; van Vugt, M.; Ammatuna, E.; van Rijn, R.S.; Vellenga, E.; van den Berg, A.; Fehrmann, R.S.N.; et al. Identification of relevant drugable targets in diffuse large B-cell lymphoma using a genome-wide unbiased CD20 guilt-by association approach. PLoS ONE 2018, 13, e0193098. [CrossRef]

5. Berry, L.D. Gould, K.L. Regulation of Cdc2 activity by phosphorylation at T14/Y15. Prog. Cell Cycle Res. 1996, 2, 99-105. 
6. Zuazua-Villar, P.; Ganesh, A.; Phear, G.; Gagou, M.E.; Meuth, M. Extensive RPA2 hyperphosphorylation promotes apoptosis in response to DNA replication stress in CHK1 inhibited cells. Nucleic Acids Res. 2015, 43, 9776-9787. [CrossRef]

7. Haferkamp, B.; Zhang, H.; Lin, Y.; Yeap, X.; Bunce, A.; Sharpe, J.; Xiang, J. BaxDelta2 is a novel bax isoform unique to microsatellite unstable tumors. J. Biol. Chem. 2012, 287, 34722-34729. [CrossRef]

8. Mattoo, A.R.; Pandita, R.K.; Chakraborty, S.; Charaka, V.; Mujoo, K.; Hunt, C.R.; Pandita, T.K. MCL-1 Depletion Impairs DNA Double-Strand Break Repair and Reinitiation of Stalled DNA Replication Forks. Mol. Cell Biol. 2017, 37. [CrossRef]

9. Megyesi, J.; Tarcsafalvi, A.; Seng, N.; Hodeify, R.; Price, P.M. Cdk2 phosphorylation of Bcl-xL after stress converts it to a pro-apoptotic protein mimicking Bax/Bak. Cell Death Discov. 2016, 2, 15066. [CrossRef]

10. Terrano, D.T.; Upreti, M.; Chambers, T.C. Cyclin-dependent kinase 1-mediated Bcl-xL/Bcl-2 phosphorylation acts as a functional link coupling mitotic arrest and apoptosis. Mol. Cell Biol. 2010, 30, 640-656. [CrossRef]

11. Zhang, M.; Dominguez, D.; Chen, S.; Fan, J.; Qin, L.; Long, A.; Li, X.; Zhang, Y.; Shi, H.; Zhang, B. WEE1 inhibition by MK1775 as a single-agent therapy inhibits ovarian cancer viability. Oncol. Lett. 2017, 14, 3580-3586. [CrossRef] [PubMed]

12. Ghelli Luserna Di Rora, A.; Beeharry, N.; Imbrogno, E.; Ferrari, A.; Robustelli, V.; Righi, S.; et al. Targeting WEE1 to enhance conventional therapies for acute lymphoblastic leukemia. J. Hematol. Oncol. 2018, 11, 99. [CrossRef] [PubMed]

13. Zhou, L.; Zhang, Y.; Chen, S.; Kmieciak, M.; Leng, Y.; Lin, H.; Rizzo, K.A.; Dumur, C.I.; Ferreira-Gonzalez, A.; Dai, Y.; et al. A regimen combining the Wee1 inhibitor AZD1775 with HDAC inhibitors targets human acute myeloid leukemia cells harboring various genetic mutations. Leukemia 2015, 29, 807-818. [CrossRef] [PubMed]

14. Klanova, M.; Andera, L.; Brazina, J.; Svadlenka, J.; Benesova, S.; Soukup, J.; Prukova, D.; Vejmelkova, D.; Jaksa, R.; Helman, K.; et al. Targeting of BCL2 Family Proteins with ABT-199 and Homoharringtonine Reveals BCL2- and MCL1-Dependent Subgroups of Diffuse Large B-Cell Lymphoma. Clin. Cancer Res. 2016, 22, 1138-1149. [CrossRef]

15. Iqbal, J.; Meyer, P.N.; Smith, L.M.; Johnson, N.A.; Vose, J.M.; Greiner, T.C.; Connors, J.M.; Staudt, L.M.; Rimsza, L.; Jaffe, E.; et al. BCL2 predicts survival in germinal center B-cell-like diffuse large B-cell lymphoma treated with CHOP-like therapy and rituximab. Clin. Cancer Res. 2011, 17, 7785-7795. [CrossRef]

16. Potter, D.S.; Letai, A. To Prime, or Not to Prime: That Is the Question. Cold Spring Harb. Symp. Quant. Biol. 2016, 81, 131-140. [CrossRef]

17. Fraser, C.; Ryan, J.; Sarosiek, K. BH3 Profiling: A Functional Assay to Measure Apoptotic Priming and Dependencies. Methods Mol. Biol. 2019, 1877, 61-76.

18. Montero, J.; Sarosiek, K.A.; DeAngelo, J.D.; Maertens, O.; Ryan, J.; Ercan, D.; Piao, H.; Horowitz, N.S.; Berkowitz, R.S.; Matulonis, U.; et al. Drug-induced death signaling strategy rapidly predicts cancer response to chemotherapy. Cell 2015, 160, 977-989. [CrossRef]

19. Ryan, J.; Letai, A. BH3 profiling in whole cells by fluorimeter or FACS. Methods 2013, 61, 156-164. [CrossRef]

20. Deng, J.; Carlson, N.; Takeyama, K.; Dal Cin, P.; Shipp, M.; Letai, A. BH3 profiling identifies three distinct classes of apoptotic blocks to predict response to ABT-737 and conventional chemotherapeutic agents. Cancer Cell 2007, 12, 171-185. [CrossRef]

21. Kim, H.Y.; Cho, Y.; Kang, H.; Yim, Y.S.; Kim, S.J.; Song, J.; Chun, K.H. Targeting the WEE1 kinase as a molecular targeted therapy for gastric cancer. Oncotarget 2016, 7, 49902-49916. [PubMed]

22. Aarts, M.; Sharpe, R.; Garcia-Murillas, I.; Gevensleben, H.; Hurd, M.S.; Shumway, S.D.; Toniatti, C.; Ashworth, A.; Turner, N.C. Forced mitotic entry of S-phase cells as a therapeutic strategy induced by inhibition of WEE1. Cancer Discov. 2012, 2, 524-539. [CrossRef] [PubMed]

23. Guertin, A.D.; Li, J.; Liu, Y.; Hurd, M.S.; Schuller, A.G.; Long, B.; Hirsch, H.A.; Feldman, I.; Benita, Y.; Toniatti, C.; et al. Preclinical evaluation of the WEE1 inhibitor MK-1775 as single-agent anticancer therapy. Mol. Cancer Ther. 2013, 12, 1442-1452. [CrossRef] [PubMed]

24. Beck, H.; Nahse-Kumpf, V.; Larsen, M.S.; O’Hanlon, K.A.; Patzke, S.; Holmberg, C.; Mejlvang, J.; Groth, A.; Nielsen, O.; Syljuåsen, R.G.; et al. Cyclin-dependent kinase suppression by WEE1 kinase protects the genome through control of replication initiation and nucleotide consumption. Mol. Cell Biol. 2012, 32, 4226-4236. [CrossRef] 
25. Beck, H.; Nahse, V.; Larsen, M.S.; Groth, P.; Clancy, T.; Lees, M.; Jørgensen, M.; Helleday, T.; Syljuåsen, R.G.; Sørensen, C.S. Regulators of cyclin-dependent kinases are crucial for maintaining genome integrity in $S$ phase. J. Cell Biol. 2010, 188, 629-638. [CrossRef]

26. Dai, J.; Luftig, M.A. Intracellular BH3 Profiling Reveals Shifts in Antiapoptotic Dependency in Human B Cell Maturation and Mitogen-Stimulated Proliferation. J. Immunol. 2018, 200, 1727-1736. [CrossRef]

27. Schuetz, J.M.; Johnson, N.A.; Morin, R.D.; Scott, D.W.; Tan, K.; Ben-Nierah, S.; Boyle, M.; Slack, G.W.; Marra, M.A.; Connors, J.M.; et al. BCL2 mutations in diffuse large B-cell lymphoma. Leukemia 2012, 26, 1383-1390. [CrossRef]

28. Adams, C.M.; Kim, A.S.; Mitra, R.; Choi, J.K.; Gong, J.Z.; Eischen, C.M. BCL-W has a fundamental role in B cell survival and lymphomagenesis. J. Clin. Investig. 2017, 127, 635-650. [CrossRef]

29. Wilson, W.H.; O'Connor, O.A.; Czuczman, M.S.; LaCasce, A.S.; Gerecitano, J.F.; Leonard, J.P.; Tulpule, A.; Dunleavy, K.; Xiong, H.; Chiu, Y.L.; et al. Navitoclax, a targeted high-affinity inhibitor of BCL-2, in lymphoid malignancies: A phase 1 dose-escalation study of safety, pharmacokinetics, pharmacodynamics, and antitumour activity. Lancet Oncol. 2010, 11, 1149-1159. [CrossRef]

30. Qi, B.; Hardwick, J.M. A Bcl-xL timer sets platelet life span. Cell 2007, 128, 1035-1036. [CrossRef] [PubMed]

31. Ford, J.B.; Baturin, D.; Burleson, T.M.; Van Linden, A.A.; Kim, Y.M.; Porter, C.C. AZD1775 sensitizes T cell acute lymphoblastic leukemia cells to cytarabine by promoting apoptosis over DNA repair. Oncotarget 2015, 6, 28001-28010. [CrossRef] [PubMed]

32. Kanfer, G.; Kornmann, B. Dynamics of the mitochondrial network during mitosis. Biochem. Soc. Trans. 2016, 44, 510-516. [CrossRef] [PubMed]

33. Taguchi, N.; Ishihara, N.; Jofuku, A.; Oka, T.; Mihara, K. Mitotic phosphorylation of dynamin-related GTPase Drp1 participates in mitochondrial fission. J. Biol. Chem. 2007, 282, 11521-11529. [CrossRef] [PubMed]

34. Qian, W.; Choi, S.; Gibson, G.A.; Watkins, S.C.; Bakkenist, C.J.; Van Houten, B. Mitochondrial hyperfusion induced by loss of the fission protein Drp1 causes ATM-dependent G2/M arrest and aneuploidy through DNA replication stress. J. Cell Sci. 2012, 125, 5745-5757. [CrossRef]

35. Kamer, I.; Sarig, R.; Zaltsman, Y.; Niv, H.; Oberkovitz, G.; Regev, L.; et al. Proapoptotic BID is an ATM effector in the DNA-damage response. Cell 2005, 122, 593-603. [CrossRef]

(C) 2019 by the authors. Licensee MDPI, Basel, Switzerland. This article is an open access article distributed under the terms and conditions of the Creative Commons Attribution (CC BY) license (http://creativecommons.org/licenses/by/4.0/). 